(C) 2019 Ye. O. KAROLINSKIY, S. O. DEMYANENKO, O. V. BIDZILYA, Yu. I. BUDASHKIN, Yu. O. GUGLYA, V. V. KAVURKA, V. G. MUSHINSKIY, O. V. ZHAKOV

\title{
ON THE FAUNA OF LEPIDOPTERA (INSECTA) OF THE NATIONAL NATURE PARK 'DVORICHANSKYI' (KHARKIV REGION, UKRAINE) AND ITS ENVIRONS. CONTRIBUTION 3
}

Каролінський, Є. О., Дем'яненко, С. О., Бідзіля, О. В., Будашкін, Ю. І., Гугля, Ю. О., Кавурка, В. В., Мушинський, В. Г., Жаков, О. В. До фауни лускокрилих (Insecta: Lepidoptera) Національного природного парку «Дворічанський» (Харківська область, Україна) та його околиць. Повідомлення 3. Вісті Харків. ентомол. m-ва. 2019. T. XXVI, вип. 1. С. $5-24$.

У статті наведено анотований список нових знахідок лускокрилих Національного природного парку «Дворічанський». Список містить 214 видів лускокрилих, з яких 174 види вперше вказуються для території парку, з них 116 видів - уперше для Харківської області, а 7 видів (Monopis pallidella, Phyllonorycter cephalariae, Amseliphora saturatella, Caryocolum petryi, Blastobasis pannonica, Neopempelia hieroglyphella, Mythimna deserticola) - уперше для України. Загалом у НПП «Дворічанський» відмічено 1238 видів Lepidoptera, з яких 11 видів занесені до Червоної Книги України та 22 види — до Червоної Книги Харківської області. 20 рис., 32 назв.

Ключові слова: Lepidoptera, лускокрилі, фауна, крейдяні відслонення, Національний природний парк «Дворічанський».

Каролинский, Е. А., Демьяненко, С. А., Бидзиля, А. В., Будашкин, Ю. И., Гугля, Ю. А., Кавурка, В. В., Мушинский, В. Г., Жаков, А. В. К фауне чешуекрылых (Insecta: Lepidoptera) Национального природного парка «Двуречанский» (Харьковская область, Украина) и его окрестностей. Сообщение 3. Изв. Харьк. энтомол. о-ва. 2019. T. XXVII, вып. 1. С. 5-24.

В статье приводится аннотированный список новых находок видов чешуекрылых Национального природного парка «Двуречанский». Список включает в себя 214 видов чешуекрылых, из которых 174 вида впервые приводятся для территории парка, из них 116 видов - впервые для Харьковской области, а 7 видов (Monopis pallidella, Phyllonorycter cephalariae, Amseliphora saturatella, Caryocolum petryi, Blastobasis pannonica, Neopempelia hieroglyphella, Mythimna deserticola) - впервые для фауны Украины. Всего в НПП «Двуречанский» отмечено 1238 видов Lepidoptera, из которых 11 видов внесены в Красную Книгу Украины и 22 вида - в Красную Книгу Харьковской области. 20 рис., 32 назв. Ключевые слова: Lepidoptera, чешуекрылые, фауна, меловые обнажения, Национальный природный парк «Двуречанский».

Karolinskiy, Ye. O., Demyanenko, S. O., Bidzilya, O. V., Budashkin, Yu. I., Guglya, Yu. O., Kavurka, V. V., Mushinskiy, V. G., Zhakov, O. V. On the fauna of Lepidoptera (Insecta) of the National Nature Park 'Dvorichanskyi' (Kharkiv Region, Ukraine) and its environs. Contribution 3. The Kharkov Entomol. Soc. Gaz. 2019. Vol. XXVII, iss. 1. P. 5-24.

An annotated list of new records of butterflies and moths of the National Nature Park 'Dvorichanskyi' is provided. The list includes 214 species, of which 174 are registered in the park for the first time, 116 are new records for Kharkiv Region, and 7 (Monopis pallidella, Phyllonorycter cephalariae, Amseliphora saturatella, Caryocolum petryi, Blastobasis pannonica, Neopempelia hieroglyphella, Mythimna deserticola) are new for Ukraine. Totally there are 1,238 species of Lepidoptera registered in the National Nature Park 'Dvorichanskyi'. There are 11 species from the Red Data Book of Ukraine and 22 species from the Red Data Book of Kharkiv Region of Ukraine registered in the park.

Keywords: Lepidoptera, butterflies and moths, fauna, limestone outcrops, National Nature Park 'Dvorichanskyi'.

20 figs, 32 refs.

I n troduction. This paper is a third contribution towards inventory of Lepidoptera of the National Nature Park (NNP) 'Dvorichanskiy' and its close environs. In our second paper (Karolinskiy et al., 2018), 1,065 species of Lepidoptera were recorded for the park. However, these data are only preliminary, and the species diversity of the study area is much higher. The examination of new material collected by us resulted in the

Karolinskiy Ye. O. Vasyl Karazin Kharkiv National University,

4, Svobody Sqr., Kharkiv, 61022, UKRAINE; e-mail: kharkov.but@gmail.com Demyanenko S. O. Severodonetsk, Luhansk Region, 93400, UKRAINE; e-mail: severlepsd@gmail.com

Bidzilya $\boldsymbol{O}$. $\boldsymbol{V}$. Institute for Evolutionary Ecology of the National Academy of Sciences of Ukraine,

37, Academician Lebedev St., Kyiv, 03143, UKRAINE; e-mail: olexbid@gmail.com

Budashkin Yu. I. T. I. Vyazemsky Karadag Scientific Station - Nature Reserve,

Kurortnoye, Feodosia, AR Crimea, UKRAINE; e-mail: budashkin@ukr.net Guglya Yu. O. Museum of Nature of the Vasyl Karazin Kharkiv National University,

8, Trinklera St., Kharkiv, 61058, UKRAINE; e-mail: pteroj@gmail.com Kavurka $\boldsymbol{V}$. V. I. I. Schmalhausen Institute of Zoology of the National Academy of Sciences of Ukraine,

15, Khmelnitskoho St., Kyiv, 01601, UKRAINE; e-mail: vitalij-kavurka@yandex.ru

Mushinskiy V. G. Zaporizhzhya, UKRAINE; e-mail: 2790601@gmail.com

Zhakov O. V. Zaporizhzhya Regional Center of Tourism and Local History, Sports and Excursion for Studying Youth,

46A, Nemirovich-Danchenko St., Zaporizhzhya, 69091, UKRAINE; e-mail: a.zhakov@gmail.com 
discovery of 174 additional species, 116 of which were recorded for the first time for the Kharkiv Region, and 7 species turned out to be new for Ukraine. We exclude 2 species, Blastobasis phycidella (Zeller, 1839) and Ancylis paludana (Barrett, 1871), from the fauna of the park due to misidentification (the correct determination is Blastobasis pannonica Šumpich et Liška, 2011 and Ancylis apicella ([Denis et Schiffermüller], 1775) resp.). Currently, the list of Lepidoptera of NNP 'Dvorichanskiy' and its environs consists of 1,238 species.

Materials and methods. This paper is based on the material collected and observed by the authors, as well as V. Kletenkin, O. Novikov and S. Tsykal, during the period from April to September 2018. We also include some previously unreported data for 2016 and 2017.

The material was observed and collected via daytime catching by a net, attracting by light $(125 \mathrm{~W}, 160 \mathrm{~W}$, and 250W mercury-vapor lamps, $8 \mathrm{~W}$ and 26W fluorescent UV lamps) and photographing.

The determination of the material was carried out by the authors. The determination by photographs was carried out only for species with distinct differences in habitus. In difficult cases, the determination was made by genitalia prepared by maceration in $8-12 \% \mathrm{NaOH}$ solution.

The material collected by V. Mushinskiy and A. Zhakov is kept by the authors; Tortricidae are in the collection of I. I. Schmalhausen Institute of Zoology of the National Academy of Sciences of Ukraine; other 'microlepidoptera' except Pyraloidea are in the collection of Institute for Evolutionary Ecology of the National Academy of Sciences of Ukraine; the rest of the material is in the collection of Museum of Nature of the Vasyl Karazin Kharkiv National University.

Places of observation, dates and collectors (for attracting by light): Novomlynsk $\left(49.88^{\circ} \mathrm{N}, 37.73^{\circ} \mathrm{E}\right)-$ further in the text 'N.' - 28-29.07.2016 (S. Demyanenko), 29.04.2017 (S. Demyanenko, Ye. Karolinskiy), 31.05.2017 (Ye. Karolinskiy), 05-06.06.2017 (S. Demyanenko, Ye. Karolinskiy), 07.06.2017 (S. Demyanenko, Ye. Karolinskiy, O. Novikov), 09.06.2017 (S. Demyanenko), 14-15.07.2017 (S. Demyanenko, Ye. Karolinskiy), 01.08.2017 (Ye. Karolinskiy), 11-12.08.2017 (S. Demyanenko), 22.08.2017 (Ye. Karolinskiy, O. Novikov), 24.08.2017 and 26.08.2017 (S. Demyanenko, Ye. Karolinskiy), 17-18.09.2017 (S. Demyanenko), 14.04.2018 (Ye. Karolinskiy, O. Novikov), 20.05.2018 (Ye. Karolinskiy, O. Novikov), 02-03.06.2018 (Ye. Karolinskiy), 15.06.2018 and 17.06.2018 (S. Demyanenko, Ye. Karolinskiy), 29-30.06.2018 (S. Demyanenko), 01.07.2018 (S. Demyanenko, Ye. Karolinskiy), 16.07.2018 (Ye. Karolinskiy), 29.07.2018 (S. Demyanenko), 21-22.09.2018 (S. Demyanenko); NNP 'Dvorichanskiy', $3 \mathrm{~km}$ NNE of Novomlynsk, 'Berezki' $\left(49.912^{\circ} \mathrm{N}, 37.756^{\circ} \mathrm{E}\right)$ - further in the text 'B.' - 04.06.2016 (V. Mushinskiy, O. Zhakov), 19-20.05.2017 (Ye. Karolinskiy, V. Kletenkin, V. Mushinskiy, O. Novikov, O. Zhakov), 17-18.06.2017 (Ye. Karolinskiy, V. Mushinskiy, O. Novikov,

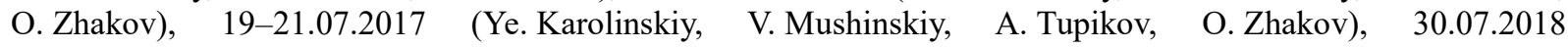
(S. Demyanenko, Ye. Karolinskiy, V. Mushinskiy, O. Novikov); NNP 'Dvorichanskiy', 4.8 km NNE of Novomlynsk, gulch 'Shlagbaumnaya' $\left(49.930^{\circ} \mathrm{N}, 37.751^{\circ} \mathrm{E}\right)$ - further in the text 'Sh.' - 31.07.2018 (S. Demyanenko, Ye. Karolinskiy, V. Mushinskiy, O. Novikov), 01.08.2018 (Ye. Karolinskiy, V. Mushinskiy); NNP 'Dvorichanskiy', $1.7 \mathrm{~km}$ NNE of Novomlynsk, gulch 'Sosnovaya' $\left(49.900^{\circ} \mathrm{N}, 37.750^{\circ} \mathrm{E}\right)$ - further in the text 'S.' - 02.07.2018 (Ye. Karolinskiy, S. Tsykal); NNP 'Dvorichanskiy', 2 km NNE of Novomlynsk, gulch 'Mogila the $\mathbf{1 s t}^{\prime}\left(49.902^{\circ} \mathrm{N}, 37.751^{\circ} \mathrm{E}\right)$ - further in the text 'M.' - 30.04 .2017 (S. Demyanenko, Ye. Karolinskiy), 07.05.2017 and 01.06.2017 (Ye. Karolinskiy), 06.06.2017 (S. Demyanenko, Ye. Karolinskiy), 03.07.2017 (Ye. Karolinskiy, O. Novikov), 06.07.2017 (Ye. Karolinskiy), 15.07.2017 (S. Demyanenko, Ye. Karolinskiy), 03.08.2017 (Ye. Karolinskiy), 26.08.2017 (S. Demyanenko, Ye. Karolinskiy), 23-24.09.2017 (Ye. Karolinskiy, O. Novikov), 15-16.04.2018 (Ye. Karolinskiy, O. Novikov), 29-30.04.2018, 19.05 .2018 and 04.06.2018 (Ye. Karolinskiy), 16.06.2018 and 01.07.2018 (S. Demyanenko, Ye. Karolinskiy), 04.07.2018, 18.07.2018, 08.08.2018, 22.08.2018 and 08.09.2018 (Ye. Karolinskiy); NNP 'Dvorichanskiy', $2.5 \mathrm{~km} \mathrm{E} \mathrm{of}$ Krasnoe Pervoe, 'Mantispovka' $\left(49.944^{\circ} \mathrm{N}, 37.796^{\circ} \mathrm{E}\right)$ — further in the text 'Mn.' - 17.07.2017 (Ye. Karolinskiy, O. Novikov).

Daytime collecting and recording were conducted mainly on the above dates (for other dates the collector's name is indicated in the text) at the following locations: right bank of Oskol River in Novomlynsk and its northern environs $\left(49.87-89^{\circ} \mathrm{N}, 37.72-74^{\circ} \mathrm{E}\right)$ - further in the text 'd-N.'; NNP 'Dvorichanskiy', right bank of Oskol River between Novomlynsk and Krasnoe Pervoe (49.89-94 $\left.{ }^{\circ} \mathrm{N}, 37.74-78^{\circ} \mathrm{E}\right)$ - further in the text 'd-N.-Kr.'; NNP 'Dvorichanskiy', right bank of Oskol River between Kamianka and Krasnoe Pervoe $\left(49.94-97^{\circ} \mathrm{N}, 37.78-83^{\circ} \mathrm{E}\right)$ - further in the text 'd-Kam.-Kr.'.

In the list below an asterisk (*) indicates a species new for NNP 'Dvorichanskiy' and its environs; two asterisks $(* *)$ - a species new for the Kharkiv region as a whole; three asterisks $(* * *)$ - a species new for Ukraine. For the species new for Ukraine, their general distribution is given. The species without asterisks were already registered in the environs of NNP 'Dvorichanskiy', i. e., in Novomlynsk and its northern surroundings (Karolinskiy et al., 2017, 2018), and now we register these species strictly at the territory of NNP. 
YE. O. KAROLINSKIY, S. O. DEMYANENKO, O. V. BIDZILYA, YU. I. BUDASHKIN,

YU. O. GUGLYA, V. V. KAVURKA, V. G. MUSHINSKIY, O. V. ZHAKOV On the fauna of Lepidoptera (Insecta) of the National Nature Park 'Dvorichanskyi' (Kharkiv Region, Ukraine) and its environs. Contribution 3

The system of Lepidoptera follows Nieukerken et al. (2011) and Heikkilä et al. (2014), and that of Coleophoridae follows Budashkin and Pusanov (2017).

R e s u l t s.

Infra or de r HE T E R O NEURA Tillyard, 1918

S uperfamily NEP T I C U L O I D A Stainton, 1854

F a m i ly N E P T I C U L I D A E Stainton, 1854

Stigmella aceris (Frey, 1857)

M a t e r i a l . d-N.-Kr., 16.06.2018 - 2 mines on Acer tataricum, 17.06.2018 - 5 mines on Acer platanoides, $01.08 .2018-$ 3 mines on Acer platanoides.

* * Stigmella trimaculella (Haworth, 1828)

M a t e ri a l. M., 03.08.2017-1 $\delta^{\text {\%. }}$

* * Stigme ll a lem niscella (Zeller, 1839)

M a t e r i a l . d-N., 29.06.2018 - 1 mine on Ulmus sp.

* Stigmella basiguttella (Heinemann, 1862)

M a t e r i a l . d-N.-Kr., 22.09.2018 - 1 mine on Quercus robur.

S uperfamily A D L O I D E A Bruand, 1850

Family A D E L I D A E Bruand, 1850

* Nemophora fasciella (Fabricius, 1775)

M a t e r i a l . d-N.-Kr., 17-18.06.2017-2 우, 16.06.2018-1 $\delta^{\text {. }}$

* * Ne mophora mi nime lla ([Denis et Schiffermüller], 1775)

M a t e r i a l. d-N.-Kr., 15.07.2017-1 ô. $^{\circ}$

* * Ade la viol el la ([Denis et Schiffermüller], 1775)

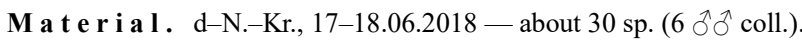

* Ade la re a m u rella (Linnaeus, 1758)

M a t e ri a l. d-N.-Kr., 29.04.2018 - 5 sp. (1 ô coll.).

* * Cauchas rufimitrella (Scopoli, 1763)

M a t e r i a l . d-N.-Kr., 13.05.2018 (O. Novikov) - $1 \mathrm{sp.}$

S u p e r f a m i ly T IS C H E R I O I D E A Spuler, 1898

F a mily T I S C H E R I I D A E Spuler, 1898

* Tischeria ekebladella (Bjerkander, 1795)

M a t e r i a l . d-N.-Kr., 22.09.2018 - 10 mines on Quercus robur.

* * Tischeria dodonaea Stainton, 1858

M a t e r i a l . d-N.-Kr., 22.09.2018 - 4 mines on Quercus robur.

* * Tischeria decidua Wocke, 1876

M a t e r i a l . d-N.-Kr., 22.09.2018 - 6 mines on Quercus robur.

* * Coptotriche angusticollella (Duponchel, [1843])

M a teria I. Sh., $31.07 .2018-1$ s. 
S u perfa mily T I N E O I D E A Latreille, 1810

F a m i ly T I N E I D A L Latreille, 1810

* * Cephimallota crassiflavella Bruand, 1850

M a te ri a l. B., $30.07 .2018-1$ औ.

* Neurothaumasia ankerella (Mann, 1867)

M a t e r i a l. M., 18.07.2018 - 1 sp.; Sh., 31.07.2018 - 2 sp.; N., 29.07.2018 - 1 sp.

* Infurcitinea albicomella (Stainton, 1851)

M a teri a l. M., 18.07.2018-1 1 .

Monopis imella (Hübner, [1813])

M a teri a I. M., 08.08.2018 - 1 sp.; Sh., 31.07.2018-1 sp.

***Monopis pallidella Zagulajev, 1955 (fig. 1-2, 15)

M a teria l. N., 15.06.2018-1 1 .

D i s t r i b u t i o n. European Central, Central Volga, Volga-Don, West Caucasus, East Caucasus, South Ural, Southeast Siberian, Pribaikal, Transbaikal, and Primorye regions of Russia, Romania and Central Asia (Baryshnikova, 2008; Petersen, Gaedike, 1996; Zagulajev, 1960).

\section{S u p e r f a mi ly G R A C I L L A R I O I D E A Stainton, 1854}

F a m i l y B U C C U L A T R I C I D A E Fracker, 1915

* Bucculatrix bechsteinella (Bechstein et Scharfenberg, 1805)

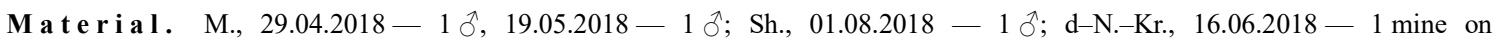
Crataegus sp.

* * Bucculatrix thoracella (Thunberg, 1794)

M a t e r i a l . d-N.-Kr., 16.06.2018 - 7 mines on Acer tataricum.

* * B u c culatrix rha m n i el la Herrich-Schäffer, 1855

M a t e r i a l. M., 15.07.2017 - 1 sp.; d-N.-Kr., 16.06.2018 - 1 mine on Rhamnus cathartica.

F a mi l y G R A C I L L A R I I D A E Stainton, 1854

* * Caloptilia fidella (Reutti, 1853)

M a t e ri a l. N., 29.07.2018-1 $\delta$.

* * Euspilapteryx a uroguttel la Stephens, 1835

M a te ri a l. Sh., 31.07.2018-1 sp.

Calybites quadrisignella (Zeller, 1839)

M a t e ria l. Sh., 31.07.2018-1 sp.

* * *Phyllonorycter cephalariae (Lhomme, 1934) (fig. 3-4, 16)

M a t e ri a l. M., 24.09.2017-1 $\delta^{\lambda}$; N., 18.09.2017-1 $\delta^{\lambda}$.

D i s t r i b u t i on. Croatia, France and Greece (De Prins W., De Prins J., 2005), Portugal and Spain (Laštůvka A., Laštůvka Z., 2014).

* Phyllonorycter issiki (Kumata, 1963)

M a t e r i a l. SE env. of Novomlynsk, 21.09.2018 - about 100 mines of various stages of development on young trees of Tilia cordata (3 mines with pupae coll., 1 औ hatched on 06.10.2018).

* * Phyllonorycter pyrifoliella (Gerasimov, 1933)

M a t e ri a l. M., 29.04.2018-1 1 . 
Superfamily Y PONOMEUTOIDEA Stephens, 1829

F a mily Y P O N O M E U T D A E Stephens, 1829

* * Paraswam merdamia nebulella (Goeze, 1783)

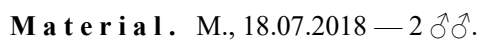

Family ARG YRESTHIIDAE Bruand, 1850

* *Argyresthia pruniella (Clerck, 1759)

M a teria l. N., 17.06.2018-1 1 .

* *Argyresthia spinosella (Stainton, 1849)

M a t e ri a l. N., 03.06.2018-1 sp.

F a mily Y P S O L O P H I D A E Guenée, 1845

* Ypsolopha vittella (Linnaeus, 1758)

M a teria l. Sh., $31.07 .2018-1 \mathrm{sp.}$

F a mily B E D E L L I I D A E Meyrick, 1880

* *Bedellia somnulentella (Zeller, 1847)

M a te ri a l. N., 22.09.2018-1 sp.

F a mily LY O N E T I I A E Meyrick, 1880

* Leucoptera malifoliella (O. Costa, 1836)

M a t e r i a l. d-N. and d-N.-Kr., 22.09.2018 - mines on Malus sp.

* * L ucoptera heringiella Toll, 1938

M a t e ri a l. B., $30.07 .2018-1$.

R e m a r ks. The second record for Ukraine. The species is not listed for Ukraine in Mey (1994, 2017),

but its type locality currently is in Ternopil region of Ukraine (Toll, 1938).

S uperfa mily D O U G L S I O I D E A Heinemann et Wocke, 1876

F a mily D O U G L A S I D A E Heinemann et Wocke, 1876

* * Tinagma ocnerostomella (Stainton, 1850)

M a teri a l. B., $18.06 .2017-1$ ㅇ.

S u perfa mily GELECHIO I D A Stainton, 1854

F a mily A U T OS T I C H I D E Le Marchand, 1947

* *Oegoconia deauratella (Herrich-Schäffer, [1854])

M a teria l. M., 18.07.2018-1 1 .

F a mily O E C O P H O R I D A E Bruand, 1850

* *Promalactis procerella ([Denis et Schiffermüller], 1775)

M a teria l. N., 30.06.2018-1 sp.

* * Metal ampra cinnamomea (Zeller, 1839)

M a te ria l. Sh., $31.07 .2018-1$ ઈै.

* * Crassa u nitella (Hübner, [1796])

M a t e r i a l. Sh., 31.07.2018 - 1 sp.; N., 30.06.2018 - 1 sp., 16.07.2018-1 sp.

Epicallima formosella ([Denis et Schiffermüller], 1775)

M a t e r i a l. M., 01.07.2018 - 1 sp., 04.07.2018 - 2 sp.; d-N.-Kr., 17.06.2018 - 1 sp. 
* * Ple urota pyropella (Linnaeus, 1767)

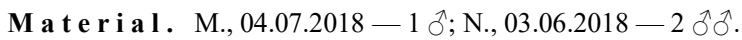

* * Pleurota aristella (Linnaeus, 1767)

M a t e ri a l. d-N., 29.06.2018 - 2 sp. (1 ô coll.).

F a m i ly D E P R E S S A R I I D A E Meyrick, 1883

S ubfamily DE P R E S A R I I N E Meyrick, 1883

* * Semioscopis steinkellneriana ([Denis et Schiffermüller], 1775)

M a t e r i a l. M., 15.04.2018-1 + , 29.04.2018-1 sp.

* * L q u etia lob ella ([Denis et Schiffermüller], 1775)

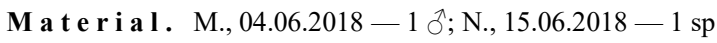

* Agonopterix cnicella (Treitschke, 1832)

M a t e ri a l. M., 08.09.2018-1 0 .

* * Agonopterix nervosa (Haworth, [1811])

M a t e r i a l. M., $16.06 .2018-1 \delta$.

* * Depressaria albipunctella ([Denis et Schiffermüller], 1775)

M a te ri a l. M., $30.04 .2018-1$.

S u b f a mi ly E T H M I N A E Busck, 1909

* *Eth mia terminella T. Fletcher, 1938

M a t e r i a l. M., 16.06.2018-3 sp.; N., 15.06.2018 - 2 sp.

F a mily COLEOPHOR I A E Bruand, 1850

* Oedicaula serinipennella (Christoph, 1872)

M a teria l. N., 09.06.2017-1 ㅇ.

* A m seliphora niveicostella (Zeller, 1839)

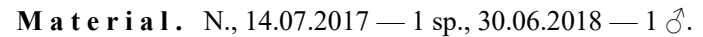

* * * Amseliphora saturatella (Stainton, 1850)

M a te ri a l. M., 04.07.2018-1 ㅇ.

D i s t r i b u t i on. Southern and Central Europe, Asia Minor, Middle East, Altai (Baldizzone, 1996; Baldizzone, Van der Wolf, Landry, 2006). All references to this species for the Volga region (Anikin, Falkovitch, 1996(1997); Anikin, 2008; Anikin, Sachkov, Zolotuhin, 2017) require the investigation of the relevant factual material, since this species was mixed with a number of other closely related species. For example, in the Crimea and the steppe zone of Ukraine ('Kamennyye Mohyly' nature reserve) these records refer to Amseliphora congeriella (Staudinger, 1859) (Bidzilya et al., 2001; Budashkin, 2004, 2013; Budashkin, Falkovitch, 2007).

* * Amseliphora coronillae (Zeller, 1849)

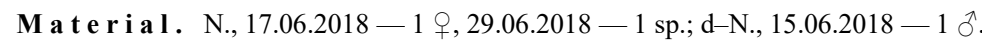

* * Multicoloria vibicella (Hübner, [1813])

M a t e r i a l. S., 02.07 .2018 - 1 sp.; d-N.-Kr., 21.05.2018 - about 10 cases on Genista sp. ( 2 cases coll., imago hatched on 14.06.2018 and 27.06.2018 (ㅇ))).

* * Multicoloria conspicuella (Zeller, 1849)

M a t e r i a l. M., 06.06.2017 - 1 sp., 06.07.2017-1 1 .

* * Multicoloria valesianella (Zeller, 1849)

M a t e r i a l. M., 16.06.2018-1 + .; N., 28-29.07.2016-1 1 .

* Damophila alcyonipennella (Kollar, 1832)

M a t e ri a l. Mn., 17.07.2017-1 ; N., 28-29.07.2016-1 9 .

* Damophila trifolii (Curtis, 1832)

M a t e r i a I. M., 24.09.2017 - 1 \%; S., 02.07.2018 - 1 sp.; N., 17.06.2018 - 1 sp. 
* * Eupista ornatipennella (Hübner, [1796])

M a te ri a I. N., 15.07.2017-1 $\delta$.

* * Eupista malatiella (Toll, 1952)

M a t e ri a l. N., $15.06 .2018-1$ \%, 17.06.2018 - $1 \mathrm{sp.}$

* * Coleophora bernoulliella (Goeze, 1783)

M a t e ri a l. N., 09.06.2017-1 sp., 15.06.2018 - 1 sp.

* *Orthographis a uricella (Fabricius, 1794)

M a t e r i a 1. M., 01.06.2017-1 ô, 01.07.2018-1 क and 1 sp.; N., 06.06.2017-1 sp.

* *Orthographis chamaedriella (Bruand, 1851)

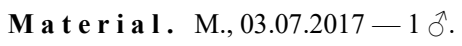

Vladdelia niveistrigella (Wocke, 1876)

M a t e ri a l. B., 30.07.2018-2 sp.; S., 02.07.2018-1 1 .

* * Casignetella obscenella (Herrich-Schäffer, [1855])

M a t e r i a l. M., 08.08.2018-1 ${ }^{\circ}, 22.08 .2018-1 \mathrm{sp.}$

* * Casignetella vestianella (Linnaeus, 1758)

M a t e ri a l. N., 28-29.07.2016-1 $\delta^{\Uparrow}$ and $1 \mathrm{sp.}$

* * Casignetella saxicolella (Duponchel, 1843)

M a te ri a l. N., 11-12.08.2017-1 $\delta, 24.08 .2017-1 \mathrm{sp.}$

* * Casignetella a mellivora (Baldizzone, 1979)

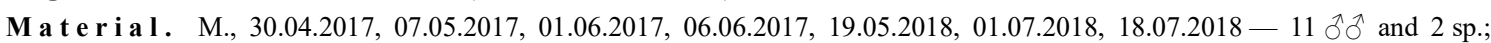
d-N.-Kr., 30.04.2017 - 1 \% .

* * Carpochena binotapennella (Duponchel, 1843)

M a t e ri a l. N., 28-29.07.2016-2 sp., 14.07.2017-1 sp.

* * Carpochena squalorella (Zeller, 1849)

M a te ri a l. N., 01.08.2017, 24.08.2017-2 sp.

* * Ionescumia clypeiferella (Hofmann, 1871)

M a t e ri a l. Sh., $01.08 .2018-1 \mathrm{sp.}$

F a mily COSM O P T E R I G I D A E Heinemann et Wocke, 1876

* * Sorhagenia rhamniella (Zeller, 1839)

M a t e ri a l. M., $04.06 .2018-1 \hat{\jmath}$ and 1 ㅇ, $16.06 .2018-1 \hat{\delta}, 18.07 .2018-1$ ㅇ.

* *Vulcaniella grandiferella Sinev, 1986

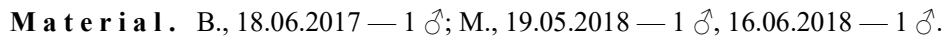

F a m i l y G E L E C H I D A E Stainton, 1854

* * Aristotelia subericinella (Duponchel, 1843)

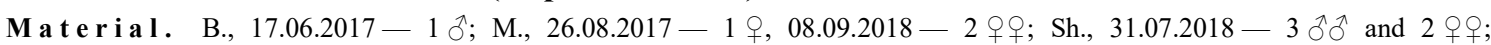
S., $02.07 .2018-1$ ठें N., 24.08.2017-1 ठิ.

* * Is ophrictis striatella ([Denis et Schiffermüller], 1775)

M a t e r i a l. M., 04.07.2018-1 ; Sh., 31.07.2018-1 .

* * Metzneria metzneriella (Stainton, 1851)

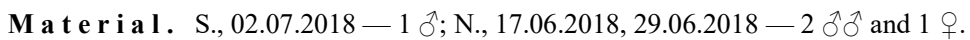

* *Monochroa sepicolella (Herrich-Schäffer, [1854])

M a teria l. M., 06.06.2017-1 $\delta$.

* * Monochroa lutulentella (Zeller, 1839)

M a te ri a l. N., $17.06 .2018-1 \delta$. 
* *Monochroa lucidella (Stephens, 1834)

M a te ria l. N., 15.06.2018-1 1 \%.

* * Bryotropha senectella (Zeller, 1839)

M a t e r i a l. N., 16.07.2018-1 $\delta^{\text {. }}$

* Re curvaria na nella ([Denis et Schiffermüller], 1775)

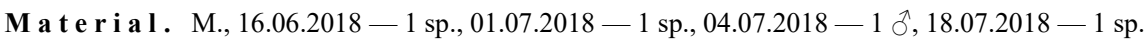

* * Te le iodes vulgella ([Denis et Schiffermüller], 1775)

M a t e r i a I. M., 04.06.2018-1 1 .

* * Carpatolechia fugacella (Zeller, 1839)

M a te ri a l. N., $30.06 .2018-1$ \%.

* * Carpatolechia notatella (Hübner, [1813])

M a t e ri a l. N., 03.06.2018-1 9 .

* * Carpatolechia aenigma (Sattler, 1983)

M a t e ri a l. Sh., 31.07.2018-2 9 .

*Gelechia scotin ella Herrich-Schäffer, [1854]

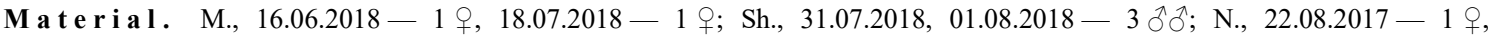
29.07.2018-1 sp.

* * Mirificarma cytisella (Treitschke, 1833)

M a te ri a l. B., $30.07 .2018-1 \mathrm{sp}$.

* Chionodes distinctella (Zeller, 1839)

M a te ri a l. N., 15.06.2018-1 $9,16.07 .2018-1$ oे.

* * Aroga flavicomella (Zeller, 1839)

M a t e ri a l. B., 19-20.05.2017-1 §.

* Athrips rancidella (Herrich-Schäffer, [1854])

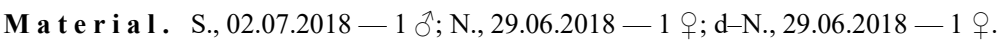

* Scrobipalpa acuminatella (Sircom, 1850)

M a teria l. B., 19-20.05.2017-1 ठै.

Scrobipalpa proclivella (Fuchs, 1886)

M a te ri a l. Sh., 31.07.2018-1 1 \%.

* * Scrobipalpa clintoni (Povolný, 1968)

M a t e ri a l. N., 24.08.2017-1 9 .

* Scrobipalpa obsoletella (Fischer von Röslerstamm, 1841)

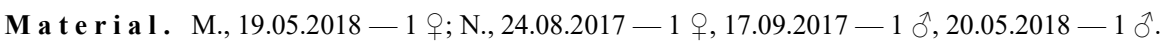

* * Scrobipalpa karadaghi (Povolný, 2001)

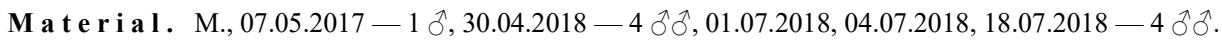

* Scrobipalpa atriplicella (Fischer von Röslerstamm, 1841)

M a t e ri a l. N., 22.08.2017-2 $\circ$ ㅇ, 24.08.2017-1 $\delta$ and 1 ㅇ.

* * Scrobipalpa bryophiloides (Povolný, 1966)

M a t e ri a l. B., $04.06 .2016-1 \hat{\delta}$.

Scrobipalpa ocellatella (Boyd, 1858)

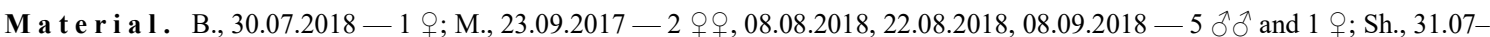
$01.08 .2018-2$ ฟैว

\section{* * Scrobipalpa samadensis (Pfaffenzeller, 1870)}

M a t e r i a l. M., 04.06.2018, 01.07.2018, 18.07.2018, 22.08.2018 - 1 o and 3 웅, 08.09.2018 - 2 우; S., 02.07.2018 - 1 웅 N., 05.06.2017-1,$+ 02.06 .2018-1$ ô. 
* * Scrobipalpula tussilaginis (Frey, 1867)

M a teria l. M., 30.04.2017-1 1 .

* * * Caryocol um petryi (O. Hofmann, 1899) (fig. 5-8, 17) and 1 \% .

M a t e r i a l. M., 04.07.2018, 08.08.2018, 22.08.2018- $1 \hat{\delta}$ and 2 우; N., 22.08.2017, 24.08.2017, 26.08.2017- $3 \hat{\delta}$

D i s t r i b u tion. Scattered records from the Pyrenees, the Alps, Germany, Hungary and some localities in northern Europe (Finland, Sweden, Latvia and Lithuania); also known from Mongolia, southern Ural Mountains and Transbaikal region of Russia (Huemer, Karsholt, 2010).

* * Aproaerema coronilella (Treitschke, 1833)

M a te ri a l. M., 24.09.2017-1 ఓో.

* * Aproaerema sangiella (Stainton, 1863)

M a teria l. N., 05.06.2017-1 §ै.

* * Aproaerema wormiella (Wolff, 1958)

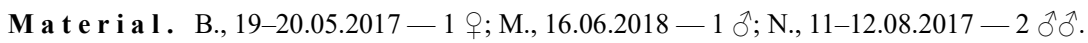

* * Aproaerema vinella (Bankes, 1898)

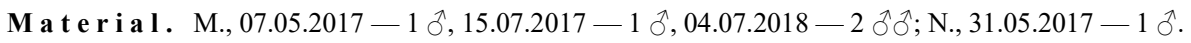

* * Aproaerema li nella (Chrétien, 1904)

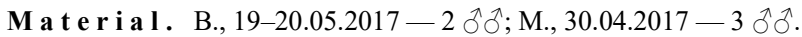

* A n arsia lineate lla Zeller, 1839

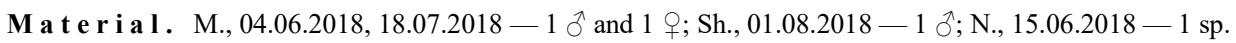

* * A compsia cinerella (Clerck, 1759)

M a t e ri a l. Sh., $01.08 .2018-1$ 万.

F a mily ELACH IS T I A E Bruand, 1850

* * Elachista obliquella Stainton, 1854

M a t e r i a l. S., 02.07.2018-1 1 .

* * Elachista dumosa Parenti, 1981

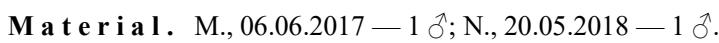

* * Elachista heringi Rebel, 1899

M a t e r i a l. M., 01.06.2017-1 $\delta^{\lambda}, 16.06 .2018-1 \delta$.

* *Elachista pollutella Duponchel, [1844]

Mat e ria l. N., 29.04.2017-2 $\widehat{\delta} \widehat{\delta}$.

* * Biselachista utonella (Frey, 1856)

M a t e r i a l. N., 29.06.2018-1 9 .

Family SCY THR I D I D A Rebel, 1901

* * Scythris seliniella (Zeller, 1839)

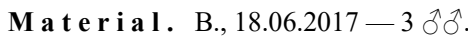

Scythris limbella (Fabricius, 1775)

M a teria l. Sh., 31.07.2018-1 sp.

* *Scythris tributella (Zeller, 1847)

M a te ri a l. B., $30.07 .2018-1 \delta$ and 2 우요.

F a mily B L A S T O B A S I D A E Meyrick, 1894

* * * B lastobas is pan no nica Šumpich et Liška, 2011 (fig. 9-10, 18)

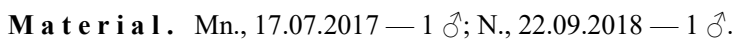


D i s t r i b u t i o n. Czech Republic, Slovakia, Hungary and Slovenia (ك̌umpich, 2011), Austria (Huemer, 2013), Orenburg province of Russia (Šumpich, Skyva, 2012).

F a m i ly M O M P H I D A E Herrich-Schäffer, 1857

* * Mompha miscella ([Denis et Schiffermüller], 1775)

M a t e ri a l. M., 19.05.2018-1 $\delta^{\text {. }}$.

* * Mompha subbistrigella (Haworth, 1828)

M a terial. N., 30.06.2018-1 1 .

S u p e r f mily P T E R P H O RO I E A Latreille, 1802

Family P T R O PHORIDAE Latreille, 1802

Oxyptilus parvidactyla (Haworth, [1811])

M a t e ri a l. Sh., $31.07 .2018-1$ §े.

* * Procapperia linariae (Chrétien, 1922)

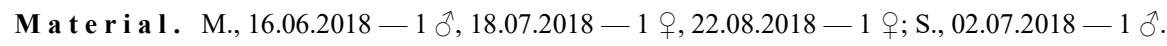

* *Procapperia maculata (Constant, 1865)

M a t e r i a I. M., 18.07.2018 - 1 ô and 1 ; B., 30.07.2018 - 1 ㅇ.

* Adaina microdactyla (Hübner, [1813])

M a t e ria l. N., 29.07.2018-1 1 .

*Hellinsia didactylites (Ström, 1783)

M a t e ri a l. S., 02.07.2018-1 1 .

* Merrifieldia tridactyla (Linnaeus, 1758)

M a t e ri a l. M., 04.06.2018-1 1 .

Su perfamily TORT R I C O I D A Latreille, 1802

Family TORTR I C I D A E Latreille, 1802

S ubfamily T ORT R I C I A E Latreille, 1802

*Acleris kochiella (Goeze, 1783)

M a t e ri a l. N., 17.06.2018-1 $\delta^{\top}$.

Phtheochroa sodaliana (Haworth, [1811])

M a te ri a l. B., 19-21.07.2017-1 1 .

Cochylimorpha straminea (Haworth, [1811])

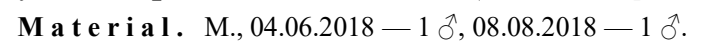

* Aethes margarotana (Clerck, 1759)

M a t e r i a l. B., 19-21.07.2017-1 o; d-N.-Kr., 29.04.2018-1 ठै.

* Aethes smeathmanniana (Fabricius, 1781)

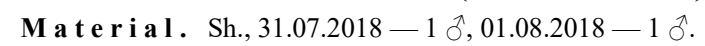

* A ethes flagellana (Duponchel, 1836)

Materia l. B., 19-21.07.2017-1 ठ‥

* * Aethes beatricella (Walsingham, 1898)

M a teria l. N., 30.06.2018-1 1 .

Aethes francillana (Fabricius, 1794)

M a t e ri a l. B., 19-21.07.2017-2 $\widehat{\jmath}$. 
Falseuncaria degreyana (McLachlan, 1869)

M a teria l. M., $30.04 .2018-1 \delta$.

* * Cnephasia genital a na Pierce et Metcalfe, 1915 M a t e ri a l. B., 19-21.07.2017-1 क; M., 04.06.2018-1 9 .

* * Oporopsamma wertheimsteini (Rebel, 1913) M a t e ri a l. M., 08.09.2018-3 sp. (1 + coll.).

Pandemis cerasana (Hübner, 1796)

M a teria l. Sh., 01.08.2018-1 0 .

Adoxophyes orana (Fischer von Röslerstamm, 1834)

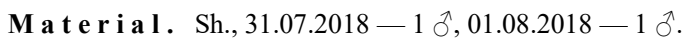

S ubfamily OLETHREUT INAE Walsingham, 1895

* Bactra lacteana Caradja, 1916

M a teria l. N., $30.06 .2018-1$ ․

*Endothenia ustulana (Haworth, [1811])

M a t e ri a l. B., $30.07 .2018-1 \delta$; S., $02.07 .2018-1$ ठै.

*Eudemis profundana ([Denis et Schiffermüller], 1775)

M a teria l. B., $30.07 .2018-1$ §.

Apotomis semifasciana (Haworth, [1811])

M a t e ri a l. M., 04.06.2018-1 $\delta$.

* Eucosma obumbratana (Lienig et Zeller, 1846)

M a teria l. N., 29.07.2018-1 §ै.

Eucosma tundrana (Kennel, 1900)

M a t e ri a l. Sh., 31.07-01.08.2018-2 sp. (1 ô coll.).

* Eucosma pupillana (Clerck, 1759)

M a t e r i a l. Sh., 01.08.2018-1 ठ; N., 29.06.2018-1 sp.

* Rhyacionia buoliana ([Denis et Schiffermüller], 1775)

M a teria l. M., $16.06 .2018-1 \delta$.

* Rhyacionia pinicolana (Doubleday, 1849)

M a te ri a l. N., 29.06.2018-1 sp.

Grapholita funebrana (Treitschke, 1835)

M a teri a l. M., 19.05.2018-1 $\delta$.

* Grapholita janthinana (Duponchel, [1835])

M a t e ri a l. S., 02.07.2018-1 $\delta$.

* Cydia a mpla na (Hübner, [1800])

M a t e ri a l. Sh., 31.07-01.08.2018-8 sp. (2 ô ô and 1 q coll.).

Superfamily COSSOIDEA Leach, 1815

Fa mily COS S I A E Leach, 1815

* A cossus terebra ([Denis et Schiffermüller], 1775)

M a teria I. N., 29.06.2018-1 sp.

F a mi ly S E S I I D A E Boisduval, 1828

* *Bembecia megillaeformis (Hübner, [1813])

M a t e r i a l . d-N.-Kr., 31.07 .2018 (V. Kletenkin) -1 q. 
* * Chamaesphecia bibioniformis (Esper, 1800) M a teria l. d-N., 29.06.2018-1 $\delta$.

S u perfa mily Z Y G A N O I D A Latreille, 1809

F a mily Z Y G A E N I A E Latreille, 1809

* Rh a gades pruni ([Denis et Schiffermüller], 1775)

M a te ri a l. d-N., 29.06.2018-1 1 .

**Zygaena punctum Ochsenheimer, 1808

M a t e r i a l. d-N.-Kr., 30.06.2018 - 1 dead sp.

Su perfamily PAP I L I O NO I D E Latreille, 1802

F a mi ly H E S P E R I I A E Latreille, 1809

* Muschampia tessell um (Hübner, [1808-1813]) M a t e ri a l. d-N.-Kr., 01.08.2018-1 §.

Fa mily P I E R I D A E Swainson, 1820

* Colias croceus (Geoffroy in Fourcroy, 1785)

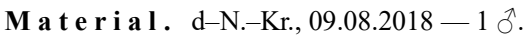

Family LYCAEN I DA E Leach, 1815

* Satyrium w-album (Knoch, 1782)

M a t e r i a l . d-N.-Kr., 16.06.2018 (D. Demchenko) -1 q.

Family NYMPHA L I A E Rafinesque, 1815

Lasiom mata maera (Linnaeus, 1758)

M a t e r i a l. d-N.-Kr., 31.07-02.08.2018 - 5 sp.

S u perfa mily P Y R A L O I D E A Latreille, 1809

F a mily P Y R A L I D A E Latreille, 1809

* Aglossa pinguinalis (Linnaeus, 1758)

M a t e ri a l. N., 01.07.2018-1 sp. indoors.

* *Hypsopygia rubidalis ([Denis et Schiffermüller], 1775)

M a t e r i a l. S., 02.07.2018-1 1 .

Galleria mellonella (Linnaeus, 1758)

M a t e ri a l. Sh., 01.08.2018-1 sp.

Epischnia illotella Zeller, 1847

M a t e ri a l. M, 22.08.2018-1 1 .

* * E legia fallax (Staudinger, 1881)

M a te ri a l. Sh., $01.08 .2018-1 \delta$.

** Neopempelia hieroglyphella (Ragonot, 1887) (fig. 11-12, 19)

M a teria l. N., $30.06 .2018-1$ \%.

D i s t r i b u t i o n. NE Iran (Ragonot, 1887; Amsel, 1954), Astrakhan province of Russia (Trofimova, 2010).

Ephestia el utell a (Hübner, [1796])

M a t e ri a l. M., 08.08.2018-1 1 , $22.08 .2018-2$ 우오. 
Hypsotropa unipunctella Ragonot, 1888

M a teria l. B., $21.07 .2017-1$ §.

F a mily CRAM B I D A E Latreille, 1810

Scoparia subfusca Haworth, [1811]

M a t e r i a l . M., 04.06.2018-2 sp. (1 ㅇ coll.), 08.09.2018-1 sp.

* * Chilo christophi Bleszyński, 1965

M a t e ri a l. B., 20.05.2017-1 ; N., 07.06.2017-1 ․

* * Euchromius gratiosella (Caradja, 1910)

M a teri a l. N., 30.06.2018-2 우요․

Crambus pratella (Linnaeus, 1758)

M a t e ri a l. M., 04.06.2018-1 sp.

Catoptria lythargyrella (Hübner, [1796])

M a t e r i a l . d-N.-Kr., 26.08.2017-1 sp.

* * A porodes floralis (Hübner, [1809])

M a t e r i a l. M., 16.06.2018-2 sp.; N., 15.06.2018-2 sp., 30.06.2018-2 sp.

* *Eurrhypis pollinalis ([Denis et Schiffermüller], 1775)

M a t e ri a l . d-N., 17.06.2018 - 1 sp., 08.09.2018 - 1 sp.

*Pyrausta purpuralis (Linnaeus, 1758)

M a teria l. N., 17.06.2018-1 sp.

Anania coronata (Hufnagel, 1767)

M a t e ri a l. Sh., $01.08 .2018-2$ sp.

Udea languidalis (Eversmann, 1842)

M a t e r i a l. M., 18.07.2018 - 1 sp.; S., 02.07.2018-2 sp.

S u perfamily B OM B Y C O I D E A Latreille, 1802

Family SPH I N G D A E Latreille, 1802

Hyles hippophaes (Esper, [1789])

M a t e ri a l. Sh., $01.08 .2018-1 \mathrm{sp.}$

S uperfamily GEOMETRO IDEA Leach, 1815

Family GEOMETRIDAE Leach, 1815

Subfamily E N OM I A E Duponchel, 1845

* * Crocallis tusciaria (Borkhausen, 1793)

M a teri a l. N., 21.09.2018-1 sp.

* Colotois pen naria (Linnaeus, 1761)

M a t e ri a l. N., 21-22.09.2018-1 $\delta^{\text {. }}$.

* Biston strataria (Hufnagel, 1767)

M a te ri a l. N., 14.04.2018-1 sp.

Cleora cinctaria ([Denis et Schiffermüller], 1775)

M a t e r i a l. M., 16.04.2018-2 sp.

S u b f a mily STERRH I A E Meyrick, 1892

* * Id a ea fuscovenosa (Goeze, 1871)

M a t e r i a l. M., 04.07.2018 - 1 sp.; d-N., 29.06.2018 - 1 sp. 
S u b f a mily LA R E T I I A E Duponchel, 1845

* * Xanthorho biriviata (Borkhausen, 1794)

M a teria l. N., 17.06.2018-1 sp.

* * Trichopteryx carpinata (Borkhausen, 1794)

M a teri a I. M., 16.04.2018-1 sp.

* * Eupithecia valerianata (Hübner, [1813])

M a te ria l. N., 17.06.2018-1 9 .

Eupithecia assimilata Doubleday, 1856

M a t e ri a l. M., 18.07.2018-1 $\delta^{\lambda}$.

S u perfa mily N O C T U O I E A Latreille, 1809

Family EREB I D E Leach, 1815

Subfamily ARCTIINAE Leach, [1815]

* Eilem a lurideola ([Zincken], 1817)

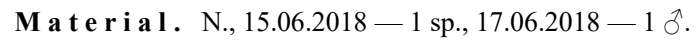

* Eilema pseudocomplana (Daniel, 1939)

M a t e r i a l. B., 30.07.2018 - 3 sp. coll.; Sh., 31.07-01.08.2017 — about 30 sp. (1 ô coll.).

Family NOCT U I A E Latreille, 1809

S ubfamily A C R O I C T I A E Heinemann, 1859

Acronicta psi (Linnaeus, 1758)

M a t e r i a l. M., 19.05.2018-1 + ; Sh., 01.08.2018-1 + .

*Acronicta leporina (Linnaeus, 1758)

M a t e ri a l. Sh., $01.08 .2018-1 \mathrm{sp.}$

S u b f a mi l y M E T O P O N I I N A E Herrich-Schäffer, [1851]

* Aegle ka ekeritziana (Hübner, [1809])

M a t e ri a l. N., 15.06.2018-1 sp.

S ubfamily A M P I P Y R I N A E Guenée, 1837

Amphipyra pyramidea (Linnaeus, 1758)

M a te ri a l. S., 02.07.2018-1 sp.; Sh., 01.08.2018-1 sp.

* A mphipyra tetra (Fabricius, 1787)

M a t e ri a l. M., 08.09.2018-1 sp.

S ubfamily X Y L E I N A E Guenée, 1837

* Caradrina albina Eversmann, 1848

M a t e ri a l. N., 22.09.2018-1 sp.

Caradrina selini Boisduval, 1840

M a t e r i a l. M., 04.06.2018-3 sp., 16.06.2018-2 sp.

*Hoplodrina superstes (Ochsenheimer, 1816)

M a t e ri a I. Sh., 31.07.2018-1 sp.

* Agrochola lota (Clerck, 1759)

M a t e ri a l. N., 21-22.09.2018-1 sp.

Eupsilia transversa (Hufnagel, 1766)

M a teria l. M., 29.04.2018 - 1 sp. 
* Apterogenum ypsillon ([Denis et Schiffermüller], 1775)

M a teria l. N., $15.06 .2018-1 \mathrm{sp.}$

* Mesogona oxalina (Hübner, [1800-1803])

M a teria l. M., 08.09.2018-1 sp.

Lenisa geminipuncta (Haworth, [1809])

M a teria l. B., 30.07.2018-2 sp.

Globia sparganii (Esper, 1790)

M a teria l. B., $30.07 .2018-1$ sp.

* Apamea oblonga (Haworth, [1809])

M a t e ri a l. N., 29.07.2018-1 sp.; B., 30.07.2018-1 sp.

*Oligia strigilis (Linnaeus, 1758)

M a teria l. N., $15.06 .2018-1$ ô.

Subfamily HADENINA E Guenée, 1837

* An arta dianthi (Tauscher, 1809)

M a te ri a I. Sh., 31.07.2018-1 sp.

* * Mythim na deserticola (Bartel, 1903) (fig. 13-14, 20)

and 1 ..

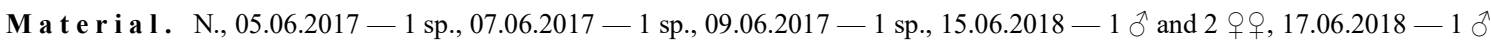

D i s t r i b u t i o n. From SE Europe (S. Ural) to Dauria and Central Mongolia, also found in the Central Asian mountain system (Hacker, Ronkay, Hreblay, 2002).

* * Mythim na alopecuri (Boisduval, 1840)

M a t e ri a l. Sh., 01.08.2018-3 sp.

Orthosia incerta (Hufnagel, 1766)

M a t e r i a l. M., 15-16.04.2018-3 sp.

* Orthosia cerasi (Fabricius, 1775)

M a t e r i a I. N., 14.04.2018-2 sp.

Orthosia gothica (Linnaeus, 1758)

M a t e ri a l. M., 15.04.2018-2 sp., 30.04.2018-1 sp.

Subfamily N OCT U I A E Latreille, 1809

* E ux o a tritici (Linnaeus, [1760]) s. 1.

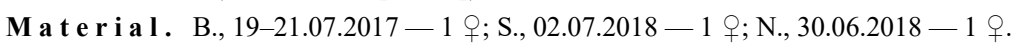

*Dichagyris signifera ([Denis et Schiffermüller], 1775)

M a t e r i a l. N., 29-30.06.2018 - 3 sp., 16.07.2018-1 sp.

Xestia baja ([Denis et Schiffermüller], 1775)

M a t e ri a l. M., 08.09.2018 - 1 sp.

* An aplectoides prasina ([Denis et Schiffermüller], 1775)

M a te ri a l. Sh., $31.07 .2018-1$ sp.

Conclusions. According to the results of our research and the literature data, 1,238 species of Lepidoptera belonging to 53 families (Table 1) are found in NNP 'Dvorichanskiy' and its environs, 174 of which are given for the park for the first time. Of these, 11 species are included to the Red Data Book of Ukraine (Akimov, 2009) and 22 species are included to the Red Data Book of Kharkiv Region of Ukraine (Tokarsky, Shandikov, Atemasova, 2013; Karolinskiy et al., 2018). The above data indicate a very high biodiversity and uniqueness of the fauna of Lepidoptera of NNP 'Dvorichanskiy'. At the same time, the list of Lepidoptera of the park is far from complete and further studies will certainly supplement it with many new species. 
Ta b l e 1. Quantitative distribution of Lepidoptera of NNP 'Dvorichanskiy' and its environs by families.

\begin{tabular}{|c|c|c|c|c|c|c|c|c|c|}
\hline Family & $\begin{array}{l}\text { Num- } \\
\text { ber of } \\
\text { species }\end{array}$ & Family & \begin{tabular}{|c|} 
Num- \\
ber of \\
species
\end{tabular} & Family & \begin{tabular}{|l|} 
Num- \\
ber of \\
species
\end{tabular} & Family & $\begin{array}{l}\text { Num- } \\
\text { ber of } \\
\text { species }\end{array}$ & Family & $\begin{array}{l}\text { Num- } \\
\text { ber of } \\
\text { species }\end{array}$ \\
\hline Hepialidae & 2 & Plutellidae & 2 & Scythrididae & 7 & Limacodidae & 1 & Lasiocampidae & 11 \\
\hline Nepticulidae & 5 & Glyphipterigidae & 2 & Blastobasidae & 1 & Zygaenidae & 12 & Brahmaeidae & 1 \\
\hline Adelidae & 7 & Ypsolophidae & 6 & Momphidae & 3 & Papilionidae & 4 & Sphingidae & 12 \\
\hline Tischeriidae & 4 & Bedelliidae & 1 & Cosmopterigidae & 11 & Hesperiidae & 12 & Geometridae & 163 \\
\hline Eriocotiidae & 1 & Lyonetiidae & 2 & Gelechiidae & 69 & Pieridae & 13 & Notodontidae & 13 \\
\hline Psychidae & 4 & Douglasiidae & 1 & Pterophoridae & 20 & Lycaenidae & 35 & Nolidae & 9 \\
\hline Tineidae & 13 & Autostichidae & 2 & Epermeniidae & 2 & Riodinidae & 1 & Erebidae & 72 \\
\hline Bucculatricidae & 3 & Oecophoridae & 10 & Choreutidae & 1 & Nymphalidae & 27 & Euteliidae & 1 \\
\hline Gracillariidae & 24 & Depressariidae & 21 & Tortricidae & 171 & Pyralidae & 76 & Noctuidae & 234 \\
\hline Yponomeutidae & 6 & Elachistidae & 6 & Cossidae & 6 & Crambidae & 91 & \multirow{2}{*}{ Total } & \multirow{2}{*}{1,238} \\
\hline Argyresthiidae & 4 & Coleophoridae & 22 & Sesiidae & 5 & Drepanidae & 6 & & \\
\hline
\end{tabular}

A cknow ledgements. The authors are sincerely grateful to Yu. Geryak (Sambir), I. Kostyuk (Zoological Museum of Taras Shevchenko National University of Kyiv), M. Kozlov (Section of Ecology, University of Turku, Finland), A. Laštůvka (Prostějov, Czech Republic), Z. Laštůvka (Mendel University, Brno, Czech Republic), V. Savchuk (Feodosia), V. Sergienko (Kyiv), S. Sinev (Zoological Institute of the Russian Academy of Sciences, St. Petersburg, Russia), J. Šumpich (National Museum, Natural History Museum, Department of Entomology, Prague, Czech Republic) for help in determining certain species, to V. Proklov (London, Great Britain) for providing rare literature sources, and to V. Kletenkin, O. Novikov, A. Tupikov, A. Velichko (NNP 'Dvorichanskiy'), D. Demchenko (Kharkiv), S. Tsykal (Kyiv) for cooperation in joint field expeditions and assistance in collecting material for this research. Yu. I. Budashkin carried out his part of the work on this paper in the framework of the implementation of the state task No. AAAA-A19-119012490044-3.

\section{R E F E R E N C E S}

Akimov, I. A., ed. 2009. Red Data Book of Ukraine. Animals [Червона книга України. Тваринний світ]. Hlobalkonsaltynh, Kyiv. ISBN: 9789669705907. [in Ukrainian].

Amsel, H. G. 1954. Die Microlepidopteren der Brandt'schen Iran-Ausbeute. 4. Teil. Arkiv för Zoologi (N. S.), 6(16), 255-326.

Anikin, V. V. 2008. Coleophoridae. In: Sinev, S. Yu., ed. Catalogue of the Lepidoptera of Russia [Каталог чешуекрылых (Lepidoptera) России]. KMK Scientific Press, St. Petersburg, Moscow, 69-82. ISBN: 9785873174577. [in Russian].

Anikin, V. V., Falkovitch, M. I. 1996(1997). On the casebearer fauna of the Lower Volga region (Lepidoptera: Coleophoridae). Zoosystematica Rossica. 5(2), 303-308. URL: https://elibrary.ru/item.asp?id=27440919.

Anikin, V. V., Sachkov, S. A., Zolotuhin, V. V. 2017. 'Fauna Lepidopterologica Volgo-Uralensis': from P. Pallas to present days. Proceedings of the Museum Witt Munich, 7. Museum Witt, Munich, Nature Research Center, Vilnius. ISBN: 9783940732309.

Baldizzone, G. 1996. Coleophoridae. In: Karsholt, O., Razowski, J., eds. The Lepidoptera of Europe: A Distributional Checklist. Apollo Books, Stenstrup, 84-94. ISBN: 8788757013.

Baldizzone, G., Van der Wolf, H., Landry, J.-F. 2006. World Catalogue of Insects. Volume 8: Coleophoridae, Coleophorinae (Lepidoptera). Apollo Books, Stenstrup. ISBN: 8788757765.

Baryshnikova, S. V. 2008. Tineidae. In: Sinev, S. Yu., ed. Catalogue of the Lepidoptera of Russia [Каталог чешуекрылых (Lepidoptera) России]. КMK Scientific Press, St. Petersburg, Moscow, 27-32. ISBN: 9785873174577. [in Russian].

Bidzilya, O. V., Budashkin, Yu. I., Zhakov, A. V., Kljuchko, Z. F., Kostjuk, I. Yu. 2001. Fauna of Lepidoptera of the 'Kamennye Mogily' Nature Reserve and its taxonomic structure [Фауна чешуекрылых (Lepidoptera) заповедника «Каменные Могилы» и её таксономическая структура]. In: Morozova, A. L., Gnyubkin, V. F., eds. Karadag. History, Biology, Archeology: the scientific works dedicated to $85^{\text {th }}$ anniversary of Karadag Scientific Station [Карадаг. История, биология, археология: сборник научньх трудов, посвящённый 85-летию Карадагской научной станции]. SONAT, Simferopol, 72-107. ISBN: 9667347729. [in Russian].

Budashkin, Yu. I. 2004. Results of the twenty-year stationary study of Lepidoptera fauna of Karadag Nature Reserve [Итоги двадцатилетнего стационарного изучения фауны чешуекрылых (Lepidoptera) Карадагского природного заповедника]. In: Morozova, A. L., Gnyubkin, V. F., eds. Karadag. History, Geology, Botany, Zoology: the scientific works dedicated to 90 ${ }^{\text {th }}$ anniversary of T. I. Vyasemsky Karadag Scientific Station and 25 th anniversary of Karadag Nature Reserve of the NAS of Ukraine [Карадаг. История, геология, ботаника, зоология: сборник научных трудов, посвящённый 90-летию Карадагской научной станции им. Т. И. Вяземского и 25-летию Карадагского природного заповедника НАН Украины], 1. SONAT, Simferopol, $323-$ 366. ISBN: 9668111281. [in Russian].

Budashkin, Yu. I. 2013. The second addition to fauna and bionomy of casebearer moth (Lepidoptera, Coleophoridae) of the Crimea [BTopoe дополнение к фауне и биологии молей-чехлоносок (Lepidoptera, Coleophoridae) Крыма]. Optimization and Protection of Ecosystems [Экосистемы, их оптимизация и охрана], 9, 3-12. URL: http://nbuv.gov.ua/UJRN/ecooo 2013 9 3. [in Russian].

Budashkin, Yu. I., Falkovitsh, M. I. 2007. Casebearers (Lepidoptera, Coleophoridae) of the Karadag Nature Reserve (South-Eastern Crimea) [Моли-чехлоноски (Lepidoptera, Coleophoridae) Карадагского природного заповедника (Юго-Восточный Крым)]. Ecosystems of the Crimea, Their Optimization and Conservation [Экосистемы Крымма, их оптимизачия и охрана], 17, 107-128. [in Russian].

Budashkin, Yu. I., Pusanov, D. V. 2017. Checklist of the casebearers (Lepidoptera, Coleophoridae) of the Crimean peninsula [Cписок молей-чехлоносок (Lepidoptera, Coleophoridae) Крымского полуострова]. Ecosystems [Экосистемы], 9, 25-36. URL: https://elibrary.ru/item.asp?id=30784179. [in Russian]. 
YE. O. KAROLINSKIY, S. O. DEMYANENKO, O. V. BIDZILYA, YU. I. BUDASHKIN,

YU. O. GUGLYA, V. V. KAVURKA, V. G. MUSHINSKIY, O. V. ZHAKOV

On the fauna of Lepidoptera (Insecta) of the National Nature Park 'Dvorichanskyi'

(Kharkiv Region, Ukraine) and its environs. Contribution 3

De Prins, W., De Prins, J. 2005. World Catalogue of Insects. Volume 6: Gracillariidae (Lepidoptera). Apollo Books, Stenstrup. ISBN: 8788757641

Hacker, H., Ronkay, L., Hreblay, M. 2002. Noctuidae Europaeae. Volume 4: Hadeninae I. Entomological Press, Sorø. ISBN: 8789430077.

Heikkilä, M., Mutanen, M., Kekkonen, M., Kaila, L. 2014. Morphology reinforces proposed molecular phylogenetic affinities: a revised classification for Gelechioidea (Lepidoptera). Cladistics, 30(6), 563-589. DOI: https://doi.org/10.1111/cla.12064.

Huemer, P. 2013. Die Schmetterlinge Österreichs (Lepidoptera): systematische und faunistische Checkliste. Tiroler LandesmuseenBetriebsgesellschaft m. b. H., Innsbruck. ISBN: 9783900083427.

Huemer, P., Karsholt, O. 2010. Microlepidoptera of Europe. Volume 6: Gelechiidae II (Gelechiinae: Gnorimoschemini). Apollo Books, Stenstrup. DOI: https://doi.org/10.1163/9789004260986.

Karolinskiy, Ye. A., Demyanenko, S. A., Zhakov, A. V., Mushinskiy, V. G. 2017. On the fauna of Lepidoptera (Insecta) of the National Nature Park 'Dvorichanskyi' (Kharkiv Region, Ukraine) and its environs [K фауне чешуекрылых (Insecta: Lepidoptera) Национального природного парка «Двуречанский» (Харьковская область, Украина) и его окрестностей]. The Kharkov Entomological Society Gazette, 25(1), 5-47. URL: http://nbuv.gov.ua/UJRN/Vkhet 2017 25 1 3. [in Russian].

Karolinskiy, Ye. A., Demyanenko, S. A., Guglya, Yu. A., Zhakov, A. V., Kavurka, V. V., Mushinskiy, V. G. 2018. On the fauna of Lepidoptera (Insecta) of the National Nature Park 'Dvorichanskyi' (Kharkiv Region, Ukraine) and its environs. Contribution 2. The Kharkov Entomological Society Gazette, 26(1), 55-114. URL: http://nbuv.gov.ua/UJRN/Vkhet_2018_26_1_6.

Laštůvka, A., Laštůvka, Z. 2014. New records of mining Lepidoptera from the Iberian Peninsula (Insecta: Lepidoptera). SHILAP Revista de lepidopterología, 42(165), 121-133. URL: https://www.redalyc.org/articulo.oa?id=45531496013.

Mey, W. 1994. Taxonomische Bearbeitung der westpaläarktischen Arten der Gattung Leucoptera Hübner, 1825, s. 1. (Lepidoptera, Lyonetiidae). Deutsche Entomologische Zeitschrift (Neue Folge), 41(1), 173-234. DOI: https://doi.org/10.1002/mmnd.19940410119.

Mey, W. 2017. Leucoptera heringiella Toll, 1938. In: Fauna Europaea (version 2017.06). URL: https://fauna-eu.org/cdm_dataportal/taxon/ 1b3633a7-8098-4237-a386-30473069b617.

Nieukerken, E. J. van, Kaila, L., Kitching, I. J., Kristensen, N. P., Lees, D. C., Minet, J., Mitter, C., Mutanen, M., Regier, J. C., Simonsen, T. J., Wahlberg, N., Yen, S.-H., Zahiri, R., Adamski, D., Baixeras, J., Bartsch, D., Bengtsson, B. Å., Brown, J. W., Bucheli, S. R., Davis, D. R., De Prins, J., De Prins, W., Epstein, M. E., Gentili-Poole, P., Gielis, C., Hättenschwiler, P., Hausmann, A., Holloway, J. D., Kallies, A., Karsholt, O., Kawahara, A. Y., Koster, J. C., Kozlov, M. V., Lafontaine, J. D., Lamas, G., Landry, J.-F., Lee, S., Nuss, M., Park, K.-T., Penz, C., Rota, J., Schintlmeister, A., Schmidt, B. C., Sohn, J.-C., Solis, M. A., Tarmann, G. M., Warren, A. D., Weller, S., Yakovlev, R. V., Zolotuhin, V. V. and Zwick, A. 2011. Order Lepidoptera Linnaeus, 1758. In: Zhang, Z.-Q., ed. Animal biodiversity: An outline of higher-level classification and survey of taxonomic richness, Zootaxa, 3148(1), 212-221. DOI: https://doi.org/10.11646/zootaxa.3148.1.3.

Petersen G., Gaedike R. 1996. Tineidae. In: Karsholt, O., Razowski, J., eds. The Lepidoptera of Europe: A Distributional Checklist. Apollo Books, Stenstrup, 31-38. ISBN: 8788757013.

Ragonot, E. L. 1887. Diagnoses d'espèces nouvelles de Phycitidae d'Europe et des Pays limitrophes. Annales de la Société Entomologique de France. Série 6, 7, 225-260. URL: https://biodiversitylibrary.org/page/8230911.

Šumpich, J., 2011. Die Schmetterlinge der Nationalparke Podyji und Thayatal. Správa Národního parku Podyjí, Znojmo. ISBN: 9788026012061.URL: https://www.np-thayatal.at/downloads_file//de/124/Schmetterlinge_Sumpich_2011_Motyli_Podyji_small.pdf.

Šumpich, J., Skyva, J. 2012. New faunistic records for a number of Microlepidoptera, including description of three new taxa from Agonoxenidae, Depressariidae, and Gelechiidae (Gelechioidea). Nota Lepidopterologica 35(2), 161-179. URL: https://www.biodiversitylibrary.org/page/46905465.

Tokarsky, V. A., Shandikov, G. A., Atemasova, T. A., eds. 2013. Red Data Book of Kharkiv Region of Ukraine. Animals [Червона книга Харківської області. Тваринний світ]. Vasyl Karazin Kharkiv National University, Kharkiv. [in Ukrainian].

Toll, S. 1938. Zwei neue Microlepidoptera-Arten aus Podolien. Annales Musei Zoologici Polonici, 13(7), 77-80.

Trofimova, T. A. 2010. New records of narrow-winged pyralids (Lepidoptera: Pyralidae: Phycitinae) of Astrakhan Province [Дополнения к фауне узкокрылых огневок (Lepidoptera: Pyralidae: Phycitinae) Астраханской области]. Entomological and Parasitological Investigation in Volga Region [Энтомологические и паразитологические исследования в Поволжье], 8, 40-42. URL: https://elibrary.ru/item.asp?id=23264787. [in Russian].

Zagulajev, A. K. 1960. Fauna of the USSR. Lepidoptera. Volume 4, issue 3. Tineidae. Part 3. Subfamily Tineinae [Фауна CССР. Насекомые чешуекрылые. T. 4, вып. 3. Настоящие моли (Тіпеidae). Ч. 3. Подсемейство Tineinae]. Publisher of the Academy of Sciences of the USSR, Leningrad. [in Russian].

Vasyl Karazin Kharkiv National University

Institute for Evolutionary Ecology of the National Academy of Sciences of Ukraine

T. I. Vyazemsky Karadag Scientific Station - Nature Reserve

Museum of Nature of the Vasyl Karazin Kharkiv National University

I. I. Schmalhausen Institute of Zoology of the National Academy of Sciences of Ukraine

Zaporizhzhia Regional Center of Tourism and Local History, Sports and Excursion for Studying Youth 

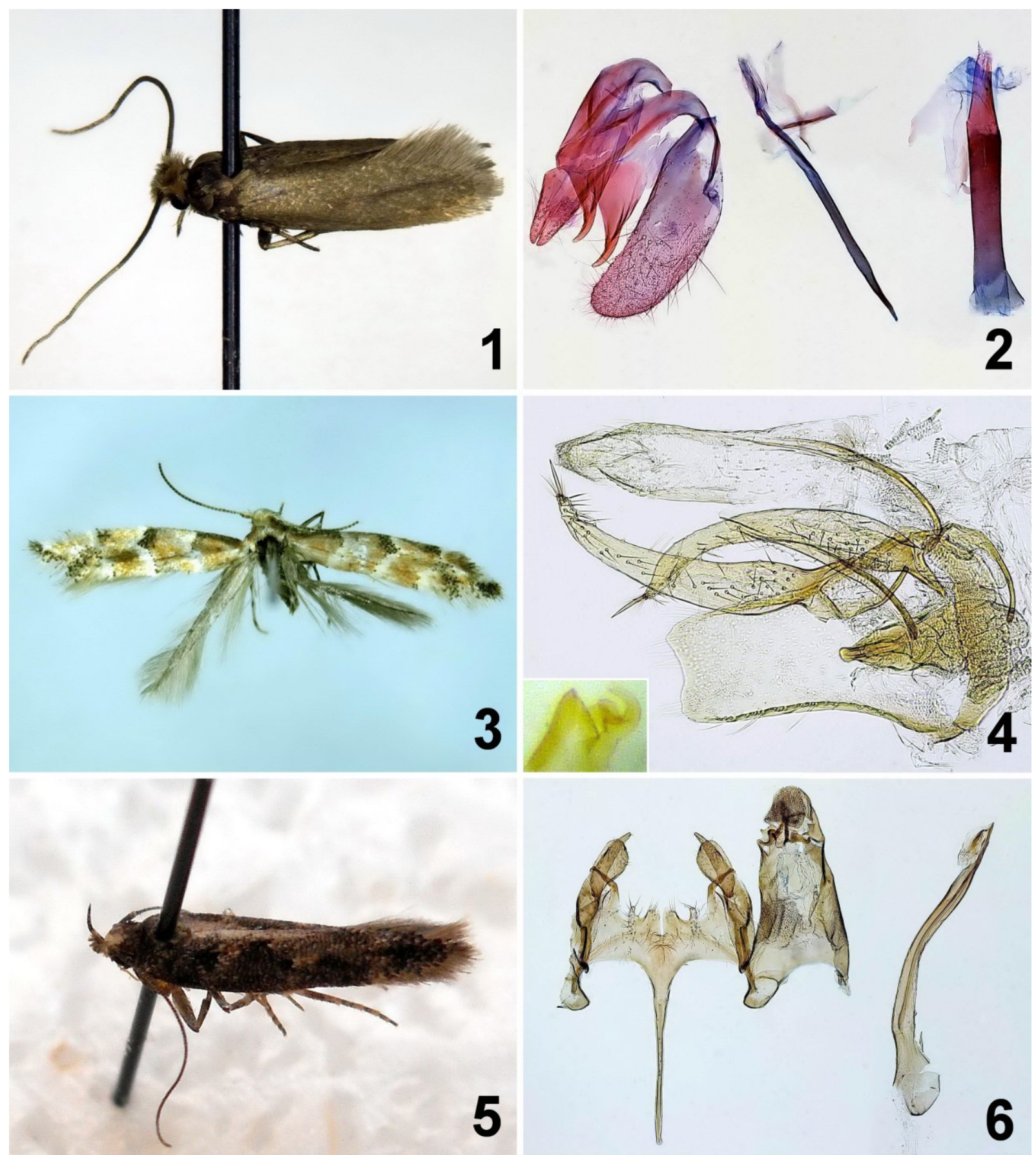

Fig. 1-6. Species of Lepidoptera new for Ukraine from NNP 'Dvorichanskiy' and its environs: 1 - Monopis pallidella, 16.06.2018, đ̂, imago; 2 - Monopis pallidella, 16.06.2018, ô, genitalia with right valva removed, saccus and aedeagus separated; 3 - Phyllonorycter cephalariae,

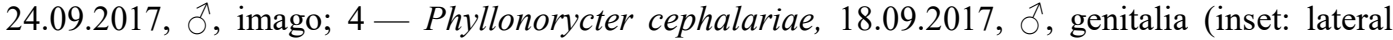
view of the end of juxta); 5 - Caryocolum petryi, 22.08.2018, ô, imago; 6 - Caryocolum petryi, 22.08.2018, ${ }^{\top}$, genitalia. 

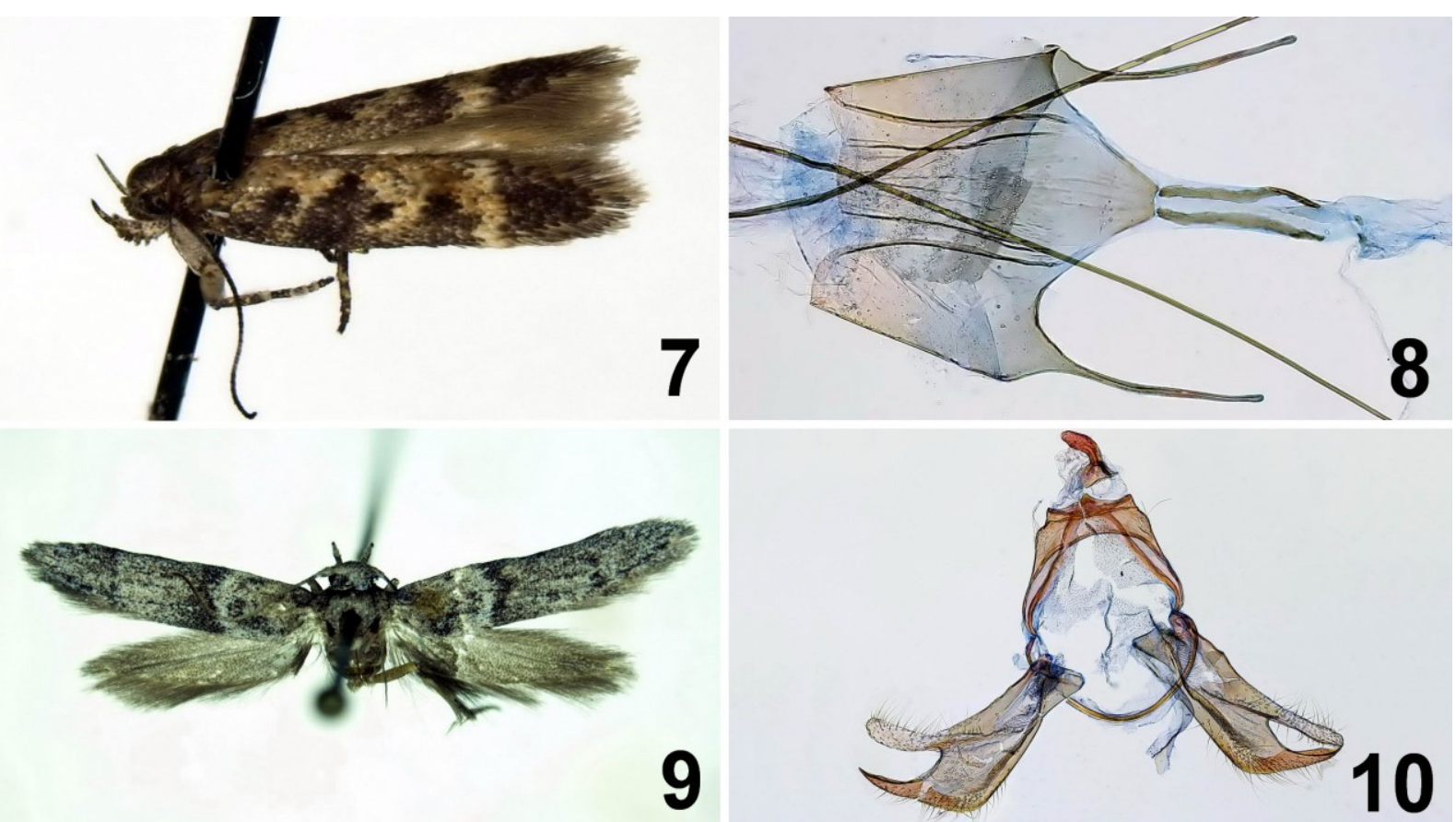

9
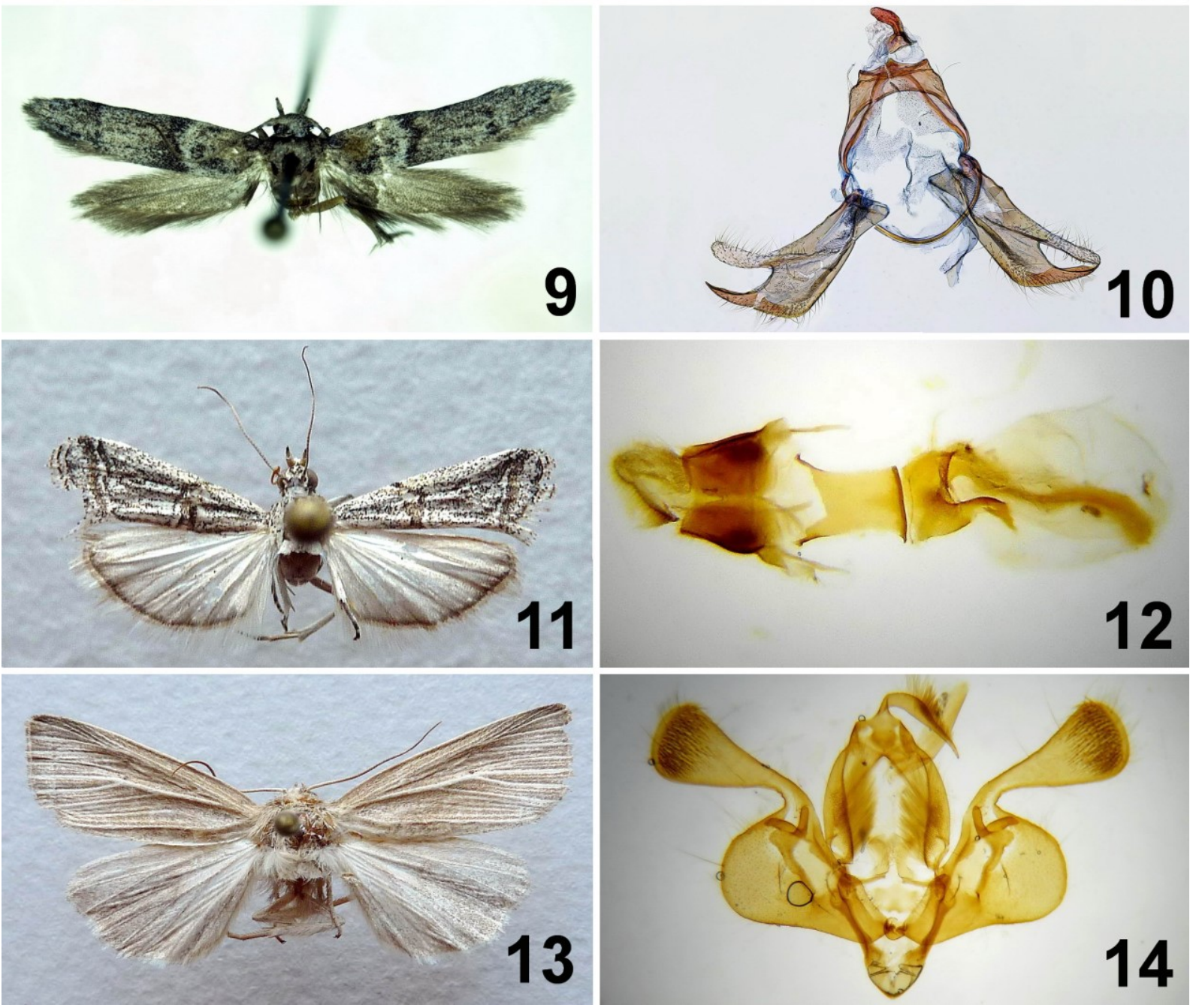

Fig. 7-14. Species of Lepidoptera new for Ukraine from NNP 'Dvorichanskiy' and its environs: 7 - Caryocolum petryi, 04.07.2018,, , imago; 8 - Caryocolum petryi, 04.07.2018,, , genitalia (segment VIII and antrum); 9- Blastobasis pannonica, 17.07.2017, ô, imago; $10-$ Blastobasis pannonica, 17.07.2017, Ô, genitalia with aedeagus removed; 11 - Neopempelia hieroglyphella, 30.06.2018, + , imago; 12 - Neopempelia hieroglyphella, 30.06.2018, + , genitalia; 13 - Mythimna deserticola, 17.06.2018, ô, imago; 14 - Mythimna deserticola,

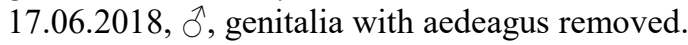



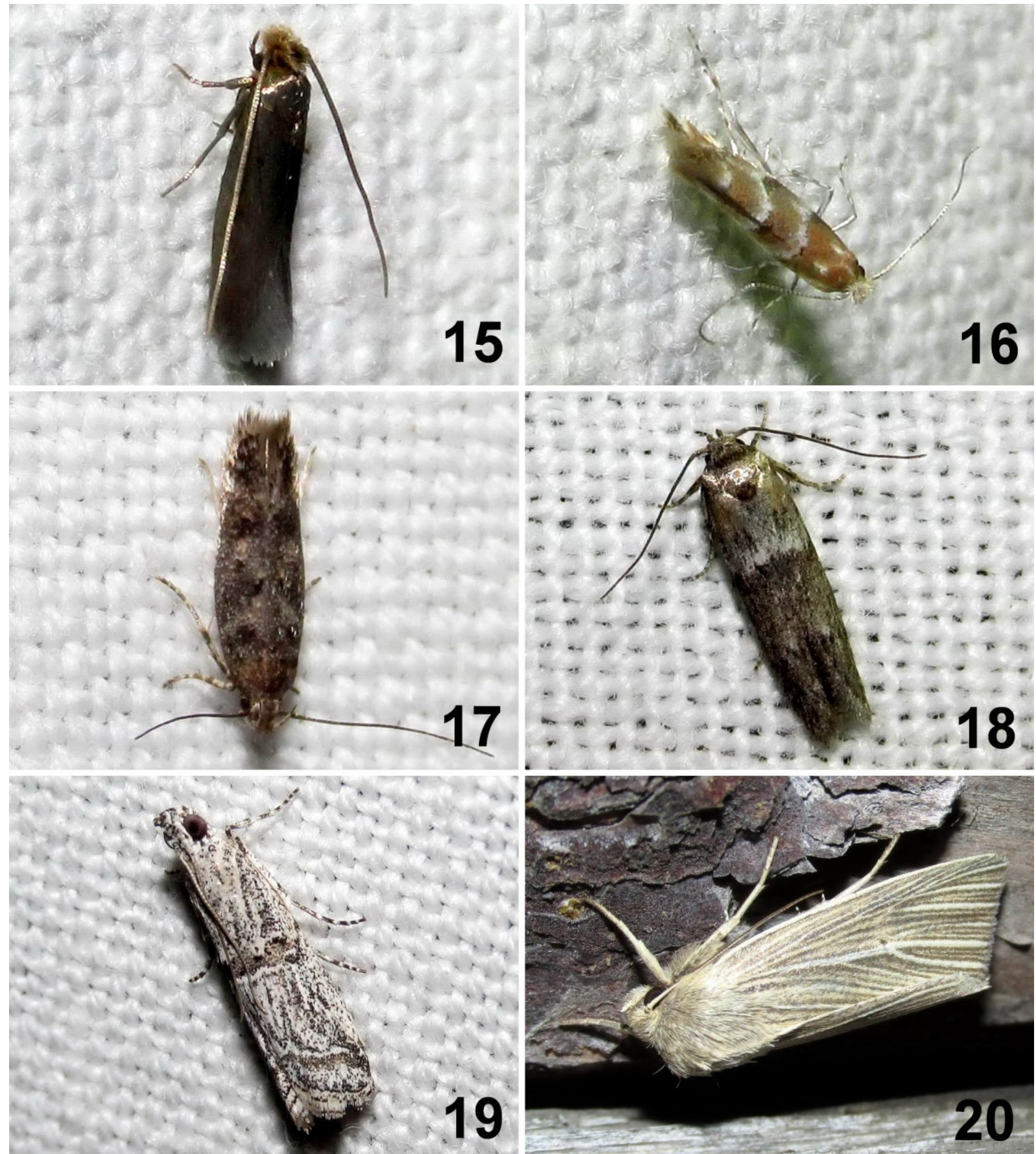

Fig. 15-2 0. Species of Lepidoptera new for Ukraine from NNP 'Dvorichanskiy' and its environs: 15 - Monopis pallidella, 16.06.2018; 16 - Phyllonorycter cephalariae, 24.09.2017; 17 Caryocolum petryi, 24.08.2017; 18 - Blastobasis pannonica, 22.09.2018; 19 - Neopempelia hieroglyphella, 30.06.2018; 20 - Mythimna deserticola, 09.06.2017. 